\title{
Mouse pregnancy-specific glycoproteins: tissue-specific expression and evidence of association with maternal vasculature
}

\author{
Freda Wynne ${ }^{1}$, Melanie Ball ${ }^{1}$, Andrew S McLellan ${ }^{1}$, Peter Dockery ${ }^{2}$, Wolfgang Zimmermann ${ }^{3}$ \\ and Tom Moore ${ }^{1}$ \\ ${ }^{1}$ Department of Biochemistry and ${ }^{2}$ Department of Anatomy, Biosciences Institute, University College Cork, \\ College Road, Cork, Ireland and ${ }^{3}$ Tumour Immunology Group, LIFE Centre, University Clinic Grosshadern, \\ Ludwig-Maximilians-University Muenchen, Marchioninistrasse 23, D-81377 Muenchen, Germany
}

Correspondence should be addressed to T Moore; Email: t.moore@ucc.ie

P Dockery is now at Department of Anatomy, National University of Ireland, Galway, University Road, Galway, Ireland

\begin{abstract}
The pregnancy-specific glycoproteins (Psg) are secreted hormones encoded by multiple genes in rodents and primates, and are thought to act as immune modulators. The only Psg receptor identified is CD9, through which Psg17 induces cytokine production from macrophages cultured in vitro. We examined temporal and spatial aspects of Psg and CD9 expression during mouse pregnancy to determine whether their expression patterns support a role in immune modulation. Using in situ hybridisation, immunohistochemistry and RT-PCR we found Psg expression in trophoblast giant cells and in the spongiotrophoblast. Psg22 is the predominant Psg family member expressed in giant cells. Detectable Psg is associated predominantly with endothelial cells lining vascular channels in the decidua, rather than with maternal immune cell markers. CD9 expression exhibited partial overlap with Psg, but without exclusive co-localisation. CD9 was observed in decidual cells surrounding early implantation sites, and in the endometrium. However, embryo transfer of wild-type embryos to CD9-deficient females indicates that maternal CD9 is not essential for successful pregnancy.

Reproduction (2006) $131721-732$
\end{abstract}

\section{Introduction}

The human pregnancy-specific glycoproteins (PSG) are the most abundant fetal proteins in the maternal bloodstream in late pregnancy (Lin et al. 1974). They are synthesised in the syncytiotrophoblast of the human placenta and in the giant cells and spongiotrophoblast of the rodent placenta (Lei et al. 1992, Rebstock et al. 1993, Kromer et al. 1996, Zhou et al. 1997). The PSG family belongs to the carcinoembryonic antigen (CEA) family, which also includes the CEA-related adhesion molecules (CEACAMs), and is part of the immunoglobulin (Ig) superfamily (Brummendorf \& Rathjen 1994). There are eleven members of the PSG family in humans that are encoded by genes clustered on chromosome 19q13.2, and seventeen mouse genes (Psg16-32) located on chromosome 7 (Thompson et al. 1990, Teglund et al. 1994, McLellan et al. 2005a). PSG proteins have a similar domain structure to the CEACAMs, but the majority lack a membrane anchor and are secreted (Teglund et al. 1994). Membrane-anchored CEACAMs are widely expressed during embryonic development and in adult tissues, and are implicated in multiple aspects of cell signalling, tissue homeostasis and disease, including carcinogenesis and regulation of immune and metabolic functions (Han et al. 2001, Zebhauser et al. 2005). PSGs and some CEACAMs are expressed almost exclusively in trophoblasts of the haemochorial placenta of rodents and primates, suggesting potential functional convergence between these otherwise divergent gene families (Rebstock et al. 1993, Zhou \& Hammarstrom 2001).

The biochemical properties and physiological functions of the members of the PSG family remain to be fully elucidated. Currently, multiple lines of evidence suggest an immunomodulatory function to prevent rejection of the allotypic fetus (Majumdar et al. 1982, Harris et al. 1984). Specifically, low PSG levels in the human maternal circulation are associated with threatened abortions, intrauterine growth retardation and fetal hypoxia, and the application of anti-PSG antibodies or vaccination with PSG induces abortion in mice and monkeys, and reduces the fertility of non-pregnant monkeys (Bohn \& Weinmann 1976, Hau et al. 1985). In addition, PSG-mediated suppression of $\mathrm{T}$ cells is correlated with increased maternal morbidity in purulent septic complications of abortion 
(Repina et al. 1989), and elevated circulating PSG levels are correlated with improved symptoms of rheumatoid arthritis (Fialova et al. 1991). Human and mouse PSGs induce secretion of anti-inflammatory cytokines from monocytes and macrophages in vitro (Wessells et al. 2000, Snyder et al. 2001), consistent with the observation of PSG-mediated switching of the immune system from a predominantly $\mathrm{TH} 1$ response to a predominantly $\mathrm{TH} 2$ response, which is considered more compatible with successful pregnancy (Motran et al. 2003).

The only PSG receptor identified to date is the integrinassociated cluster of differentiation 9 antigen (CD9) receptor. In macrophages it was found to bind the N1 domain of both Psg17 and Psg19 (Waterhouse et al. 2002). The interaction of Psg17 and CD9 was found to be necessary for the induction of secretion of anti-inflammatory cytokines (Ha et al. 2005). Psg17 has also been shown to prevent sperm-egg fusion by interrupting the binding of CD9 to a ligand on the egg surface (Ellerman et al. 2003). No receptor for human PSG has been identified and, unlike mouse Psg17, human PSG do not require CD9 to induce cytokine production from mouse macrophages (Ha et al. 2005).

CD9 is a tetraspanin, which is an integral membrane protein with four transmembrane domains and two extracellular domains. Tetraspanin family members have been implicated in a variety of cellular and physiological processes, such as cell motility, aggregation, signalling, and fusion (Boucheix \& Rubenstein 2001). Tetraspanins are believed to act as 'molecular facilitators', grouping together cell-surface proteins and thus increasing the formation and stability of functional protein complexes (Maecker et al. 1997). CD9 associates with a great variety of membrane proteins, such as membrane anchored growth factors, integrins, members of the immunoglobulin superfamily and other tetraspanins (Boucheix \& Rubenstein 2001). Although little is known about the role of CD9 in reproduction, human CD9 may function in extravillous trophoblast invasion of maternal tissues (Hirano et al. 1999). CD9 expression has not been analysed in mouse pregnancy, and it is not known whether PSG and CD9 expression patterns overlap in vivo.

In this study we examined the expression of Psg and CD9 during mouse placental development, and we investigated whether CD9 is necessary for successful pregnancy. Our results provide evidence of tissue-specific regulation of mouse Psg genes, and a possible association of secreted Psg protein with vascular endothelium.

\section{Materials and Methods}

\section{Mice}

Mouse tissues were obtained from the Biological Services Unit, University College Cork. Mouse strains used were $\mathrm{CD} 1, \mathrm{C} 57 \mathrm{Bl6} / \mathrm{J}, \mathrm{B}_{6 \mathrm{CBF}}$, and the CD9 null mutant on the C57Bl6/J background (hereafter CD9), which was obtained from Dr Claude Boucheix, Institut National de la
Santé et de la Recherche Médicale (INSERM), unite 268, Hopital Paul-Brousse, 94800 Villejuif, France. Embryonic (E) stage refers to the gestational age of the embryo. The morning on which the vaginal plug was found is counted as day one (E1) of gestation.

\section{Production of recombinant baculovirus mouse Psg21-V5/His and human PSG1-V5/His}

Recombinant Psg21 and PSG1 proteins were produced using the baculovirus protein expression system (Invitrogen Life Technologies).

\section{Subcloning in pBlueBac4.5V5/His}

Psg21wt was cloned previously into pcDNA3.1 (Ball et al. 2004). The Psg21 wt open reading frame (ORF) was reamplified by PCR, using primers incorporating restriction enzyme sites at each end (Xhol at $5^{\prime}$ and EcoRI at $3^{\prime}$ ) to allow ligation into pBlueBac4.5V5/His (Invitrogen Life Technologies) in frame with the $\mathrm{V} 5 / \mathrm{His}$ tag. A vector with a carboxyl (C) terminal $\mathrm{V} 5 / \mathrm{His}$ tag was used to prevent interference of the tag with putative Psg functional domains at the $\mathrm{N}$ terminus. The human PSG1a ORF was amplified by PCR from an existing cDNA clone (Zimmermann et al. 1989).

\section{Production of viral DNA}

Recombinant plasmid and Bac-N-Blue DNA (Invitrogen Life Technologies) were co-transfected into adherent Spodoptera frugiperda-9 (Sf9) cells in Grace's Insect media (Invitrogen Life Technologies). Cultures were closely observed for signs of viral infection and serial dilutions of the infected culture media were then used for plaque assay. Blue plaques representing sites of recombinant viral infection were picked and used to infect Sf9 s in adherent culture. The cultures were observed for signs of recombinant only viral infection (seen as absence of occlusion bodies $(\mathrm{OCC}-))$. The cell suspensions from OCC - wells were split into two fractions, one for PCR analysis to confirm absence of wild-type baculovirus and the other to be kept as the initial (P1) viral stock. The P1 viral stock of a recombinantonly culture was then used to generate a small-scale high titre (P2) viral stock, which was then used to generate a large-scale, high titre stock (P3). Plaque assay was then carried out to determine the titre of the $\mathrm{P} 3$ viral stock.

\section{Protein production and purification}

Small-scale protein expression was performed to establish the optimal multiplicity of infection (MOI) and time course for expression of the recombinant Psg21 and PSG1 protein in Sf9 in suspension in serum-free medium (SF900 II, Invitrogen Life Technologies). Recombinant protein production was determined by Western blot with antibody targeted against the V5 epitope tag. Large-scale expression was carried out in one-litre spinner flasks using the $\mathrm{MOI}$ and time course determined previously, in this case, an MOI of 
3 phage particles per Sf9 cell and time course of 5 days in SF900 II. After 5 days culture the cells were spun out and the Xpress Protein Purification system (Invitrogen Life Technologies) was used to purify the recombinant protein from the medium. The medium was batch bound to the ProBond nickel chelating resin (Invitrogen Life Technologies), the resin was allowed to settle and the supernatant drained off. The resin was then washed, and bound protein was eluted using increasing concentrations of imidazole. The eluate was collected in $1 \mathrm{ml}$ fractions and analysed for recombinant protein by spectrophotometer and Western blot. Fractions with contaminating proteins were pooled and subjected to a second round of purification on the ProBond resin. All clean fractions of recombinant protein were pooled and dialysed against $50 \mathrm{mM}$ Tris $\mathrm{pH} 7.5$, and then further purified by anion exchange chromatography using an increasing step gradient of $\mathrm{NaCl}$ in $50 \mathrm{mM}$ Tris $\mathrm{pH}$ 7.5. The clean fractions were then pooled, dialysed against phosphate buffered saline $\mathrm{pH} 7.5$ (PBS) and concentrated using centrifuge concentrators (Millipore, Ireland BV, Cork, Ireland). Concentrated protein was used for polyclonal antibody production.

\section{Production of polyclonal antisera to Psg21 and PSG1}

\section{Antiserum production}

Preimmune bleeds were taken prior to inoculation of rabbits with recombinant Psg21 and PSG1 proteins. An initial injection of $500 \mu \mathrm{g}$ recombinant protein was administered with Freund's Complete Adjuvant, followed by four booster doses of $250 \mu \mathrm{g}$ protein with Freund's Incomplete Adjuvant at 3-week intervals. Test bleeds were taken 10 days after each injection and tested on Western blot against recombinant baculovirus Psg21 and PSG1. A final bleed from each rabbit was taken by exsanguination.

\section{Antiserum validation}

Polyclonal antisera were tested against recombinant baculovirus Psg21 and PSG1, placental tissue homogenates from pregnant and non-pregnant mice, or tissue homogenates from human term placentas or maternal pregnant serum, by Western blotting.

\section{Immunohistochemistry and immunofluoresence}

\section{Tissue preparation}

Tissues were collected from non-pregnant and pregnant mice at varying stages of pregnancy, washed in cold $\left(4^{\circ} \mathrm{C}\right)$ PBS, embedded in OCT cryopreservation compound (BDH Laboratory Supplies, Poole, UK) and frozen in an iso-pentane bath in liquid nitrogen or at $-80^{\circ} \mathrm{C}$. Frozen tissue blocks were stored at $-80^{\circ} \mathrm{C}$ until use. Sections $(5 \mu \mathrm{m}$ thick) were cut and mounted on Superfrost Plus microscope slides (BDH Laboratory Supplies), air dried for 15$30 \mathrm{~min}$, fixed in cold $\left(4^{\circ} \mathrm{C}\right)$, fresh $4 \%$ paraformaldehyde in PBS for $10 \mathrm{~min}$ and then rinsed in cold PBS. The slides were then equilibrated in Tris-buffered saline at room temperature (TBS, $\mathrm{pH}$ 7.4) for $5 \mathrm{~min}$. Sections for immunohistochemistry were treated for endogenous peroxidase activity by incubating in $2 \%$ hydrogen peroxide in TBS for $30 \mathrm{~min}$.

\section{Immunohistochemistry}

Immunohistochemistry (IHC) was performed using the Vectastain Elite ABC system (Vector Laboratories Ltd, Peterborough, UK). For immunohistochemical localisation of Psg, sections were blocked with $5 \%$ non-fat milk in TBS containing $0.1 \%$ Triton-X 100 (TBS-Tx) for $1 \mathrm{~h}$ at room temperature (RT) and then incubated with primary antiserum or preimmune serum (Psg21 1:500, preimmune serum 1:500) in blocking buffer overnight at $4{ }^{\circ} \mathrm{C}$. Sections were then washed three times for $10 \mathrm{~min}$ in TBS-Tx before incubating with biotinylated anti-rabbit secondary antibody (Vector Laboratories, 1:200), washed again three times for 10 min in TBS-Tx and detected using the ABC system and Vector VIP peroxidase substrate kit (Vector Laboratories), following the manufacturer's instructions. Optimisation of different blocking buffers showed that non-fat milk gave the best results; negative controls were therefore needed in all experiments to ensure endogenous biotin in the milk did not interfere with the $A B C$ system. To avoid possible variation in biotin levels from the non-fat milk, TBS-Tx non-fat milk was prepared in bulk, aliquoted and frozen at $-20^{\circ} \mathrm{C}$ and used for all procedures. Following $\mathrm{IHC}$, sections were counterstained with Harris' haematoxylin $(\mathrm{BDH})$, differentiated, dehydrated, and mounted with DePeX (BDH) permanent mounting medium.

Immunohistochemical staining with a rat anti-mouse CD9 antibody (RDI-MCD9-C8, Research Diagnostics Inc., Concord, MA, USA) was carried out using the Vector Mouse on Mouse (MOM) kit (Vector Laboratories) following the manufacturer's instructions, to minimise cross reactivity between the rat secondary antibody and mouse tissues. Briefly, sections were blocked overnight with MOM IgG blocking solution in TBS-Tx at $4{ }^{\circ} \mathrm{C}$, washed twice for 5 min with TBS-Tx, incubated with MOM solution in TBS-Tx for $15 \mathrm{~min}$, incubated with anti-CD9 antibody at a dilution of 1:1000 for $1 \mathrm{~h}$ at RT, washed three times for 10 min in TBS-Tx, incubated with secondary biotinylated anti-rat (Vector Laboratories) at 1:1000 for $1 \mathrm{~h}$ then washed, detected and mounted as for Psg. An anti-Ceacam antibody, AgB10 (Kuprina et al. 1990), was used at a dilution of 1:50 in conjunction with the MOM kit as for CD9.

\section{Immunofluorescence}

Single antibody immunofluorescence was carried out using the same basic procedure as for immunohistochemistry except fluorescent secondary antibodies were used instead of the $\mathrm{ABC}$ system. Donkey anti-rabbit rhodamine (Abcam Plc, Cambridge, UK) for Psg was used at a concentration of 1:100 and sheep anti-rat FITC (Abcam) for CD9 at a concentration of 1:100. After incubation with secondary 
antibody, sections were washed three times for $10 \mathrm{~min}$ with TBS-Tx, once for $5 \mathrm{~min}$ in PBS, post fixed with cold $4 \%$ paraformaldehyde in PBS for $10 \mathrm{~min}$, washed twice for $5 \mathrm{~min}$ in PBS and then mounted with Vectashield Mounting medium with DAPI (Vector Laboratories).

Double immunofluorescence was carried out sequentially due to the incompatibility of the rabbit (for Psg) antibodies with the MOM kit required for the rat antibodies (for CD9 and CD31). Psg staining was carried out first, followed by CD9 or CD31. The anti-CD31 antibody (Abcam) was used at 1:20, with blocking and washing steps as for the anti-CD9 antibody.

\section{In situ hybridisation}

In situ hybridisation was carried out using digoxigeninlabelled RNA probes. A 252 bp fragment was amplified from the Psg21 A domain incorporating EcoRI restriction sites at the $5^{\prime}$ and $3^{\prime}$ ends (forward (F) 5'GCGGAATTCTGTTCAAGTCAACATCTACAAGC; reverse (R) 5'CGCGAATTCGGGTTGAAGGCCTCACATT). The resulting PCR fragment was ligated into the PSPT18 multiple cloning site at the EcoRI site. Two constructs were created with the insert in opposite orientations for generating sense and antisense probes. RNA probes were synthesised in the presence of digoxigenin-labelled UTP using T7 polymerase. In situ hybridisations were carried out on $5 \mu \mathrm{m}$ thick cryosections mounted on Superfrost Plus microscope slides (BDH). All solutions were prepared using diethylpyrocarbonate-treated water or RNase-free molecular biology grade water (Sigma); all glassware, bench tops and equipment were treated with RNase Away (Invitrogen Life Sciences) to remove RNase contamination prior to each experiment. Sections were treated as follows: air dried for $30 \mathrm{~min}$, fixed in cold (4\%) paraformaldehyde in PBS for $10 \mathrm{~min}$, rinsed three times for $5 \mathrm{~min}$ in TBS, $200 \mathrm{mM} \mathrm{HCl}$ for $10 \mathrm{~min}, 0.5 \%$ acetic anhydride in $100 \mathrm{mM}$ Tris for $10 \mathrm{~min}$, rinsed in TBS for $5 \mathrm{~min}$, baked on a hotplate at $95^{\circ} \mathrm{C}$ for $4 \mathrm{~min}$, rinsed in TBS and then incubated at $55^{\circ} \mathrm{C}$ with hybridisation buffer for $1 \mathrm{~h}$. Sections were then incubated with $0.1 \mathrm{ng} / \mu \mathrm{l}$ probe in hybridisation buffer at $55^{\circ} \mathrm{C}$ in a humid chamber for $2 \mathrm{~h}$. Unbound probe was removed by washing twice for $5 \mathrm{~min}$ in $4 \times \mathrm{SSC}$ at RT, three times for $5 \mathrm{~min}$ in $60 \%$ deionised formamide/ $0.2 \times \mathrm{SSC}$ at $55^{\circ} \mathrm{C}$, twice for $5 \mathrm{~min}$ in $2 \times \mathrm{SSC}$ at $37^{\circ} \mathrm{C}$, two times for $10 \mathrm{~min}$ in $0.1 \times \mathrm{SSC}$ at $37^{\circ} \mathrm{C}$. Sections were then equilibrated in TBS and blocked overnight at $4{ }^{\circ} \mathrm{C}$ with TBS-Tx containing $2 \%$ normal sheep serum (NSS). For immunological detection of bound digoxigenin (DIG) probe, anti-DIG antibody (Roche) was used at a dilution of 1:700 in TBS-Tx NSS for two hours at room temperature. Sections were washed twice for $10 \mathrm{~min}$ in TBS and then either detected using Vector Red alkaline phosphatase substrate kit (Vector Laboratories) following the manufacturer's instructions and counterstained with haematoxylin, or detected using NBTBCIP and the DIG Nucleic Acid detection kit (Roche) following the manufacturer's instructions.

\section{CD9 expression analysis of maternal tissues}

Decidual tissue was dissected from implantation sites of pregnant CD1 mice at E8, E9, E10 and E11 along with the corresponding uterine tissues. Lung, liver and brain from an E10 pregnant mouse were also included. Tissues were homogenised in lysis buffer $(0.1 \mathrm{M}$ Tris- $\mathrm{HCl} \mathrm{pH} 8,0.1 \%$ Triton-X 100) containing protease inhibitors (phenylmethylsulphonyl fluoride $100 \mu \mathrm{g} / \mathrm{ml}$, leupeptin $0.5 \mu \mathrm{g} / \mathrm{ml}$, aprotinin $0.5 \mu \mathrm{g} / \mathrm{ml}$, pepstatin A $1 \mu \mathrm{g} / \mathrm{ml}$ ), insoluble debris was removed and the supernatants quantified and used for SDSPAGE and Western blotting analysis. SDS-PAGE (for Western blotting) for CD9 was carried out under non-reducing conditions, with $15 \mu \mathrm{g}$ protein of each sample being heat denatured at $95^{\circ} \mathrm{C}$ for $10 \mathrm{~min}$ in a non-reducing loading buffer and resolved on a $5 \%$ stacking/15\% resolving gel. A duplicate gel was set up, as for CD9, for the $\beta$-actin loading control but run under reducing conditions. The gels were then electrotransferred to nitrocellulose membrane and immunodetection was carried out. Briefly, the blots were blocked with $5 \%$ non-fat milk in phosphate buffered saline $0.1 \%$ Tween 20 (PBS-T) for $1 \mathrm{~h}$ at RT, incubated with primary antibody overnight at $4{ }^{\circ} \mathrm{C}$, washed three times for 10 min in PBS-T, incubated for $1 \mathrm{~h}$ with secondary horseradish peroxidase (HRP)-conjugated antibody in PBS-T, washed three times in PBS-T for $10 \mathrm{~min}$ and then detected using Supersignal West Pico Chemiluminescent Substrate (Pierce Biotechnology Inc, Rockford, IL, USA) and exposed to Kodak X-OMAT AR film (Sigma). Unless stated otherwise incubations and washes were carried out at room temperature and on a rocker. The primary antibodies, CD9 (used previously) and $\beta$-actin (Sigma), were used at a dilution of 1:1000 in blocking buffer. The secondary antibodies, antirat-HRP (Sigma) for CD9 and anti-mouse-HRP (Sigma) for $\beta$-actin, were used at a dilution of 1:20000 in PBS-T.

\section{Psg gene expression analysis in trophoblast giant cells}

Placentas from CD1 mice were collected at E8, E9, E10, E11, and primary trophoblast giant cells were dissected from the surrounding tissue. For each developmental stage, cDNA was analysed from two litters (cDNA was pooled from approximately six placentas per litter). Extracted tissue was homogenised in $1 \mathrm{ml}$ TRI-reagent (Sigma-Aldrich) and total RNA was isolated. First-strand cDNA was synthesised using $1 \mu \mathrm{g}$ total RNA as template in a $20 \mu$ l reaction using random hexamer priming and Moloney-murine leukaemia virus reverse transcriptase (Invitrogen).

\section{Quantitative RT-PCR}

Primers were designed that amplify all known mouse $P s g$ gene sequences: PsgF: 5'-TYCAYCCDKTGGHTCTTCAAYA; PsgR: 5'-CACAYYGRTAMTYTCCASCATC. Normalisation of expression level to the housekeeping gene, hypoxanthineguanine phosphoribosyl transferase (Hprt), was used to avoid discrepancies caused by variations in input RNA or in reverse transcription efficiencies. The following primer 
sequences were used: Hprt forward (HprtF): 5'-CTCATGGACTGATTATGGACAGGAC; Hprt reverse (HprtR): 5'-GCAGGTCAGCAAAGAACTTATAGCC. Quantitative PCR was performed using the ABI PRISM 7900 sequence detection system (SDS) and the SYBR GREEN qPCR kit (Applied Biosystems, Foster City, CA, USA). The SYBR GREEN PCR master mix consists of Amplitaq Gold DNA polymerase, optimised PCR buffer, $25 \mathrm{mM} \mathrm{MgCl}$, dNTP mix and AmpErase UNG. PCR amplifications were performed in a total volume of $15 \mu \mathrm{l}$ in duplicate wells.

The following PCR protocol was used: denaturation program $\left(95^{\circ} \mathrm{C}\right.$ for $\left.10 \mathrm{~min}\right)$, amplification and quantification program repeated for 40 cycles $\left(95^{\circ} \mathrm{C}\right.$ for $15 \mathrm{~s}, 55^{\circ} \mathrm{C}$ for $30 \mathrm{~s}, 72^{\circ} \mathrm{C}$ for $45 \mathrm{~s}$ with a single fluorescence measurement), melting curve program $\left(60^{\circ} \mathrm{C}-95^{\circ} \mathrm{C}\right.$ with a heating rate of $1{ }^{\circ} \mathrm{C}$ per $30 \mathrm{~s}$ and a continuous fluorescence measurement). Thereafter, PCR products were identified by generating a melting curve, which was also used to assess the occurrence of putative PCR artefacts (primer-dimers) or non-specific PCR products. The sizes of the RT-PCR products were confirmed by gel electrophoresis on a standard $1.5 \%$ agarose gel stained with ethidium bromide and visualised by exposure to ultraviolet light. Results were described as mean Psg expression relative to mean Hprt expression.

\section{Identification of Psg gene transcripts in trophoblast giant cells}

To identify Psg genes expressed in giant cells, PsgF and PsgR primers previously described were used to amplify Psg cDNA transcripts present $\left(P_{s g} 22\right.$ and $P_{s g} 25$ are of identical sequence in the amplicon generated by these primers). PCR was performed in $50 \mu \mathrm{l}$ using $0.4 \mathrm{U}$ Accuzyme (Bioline, London, UK) according to the manufacturer's instructions. RT-PCR products were purified using the Qiaquick PCR purification kit (Qiagen, Crawley, West Sussex, UK). Purified amplicons were subcloned into pSTblue-1 vector and transformed into NovaBlue Singles competent cells (Novagen, EMD Bioscience, Madison, MI, USA). Positive clones were identified by a diagnostic PCR screen of bacterial colonies. For each developmental stage tested eight clones per litter were bi-directionally sequenced (Macrogen Inc, Gasan-Dong, Gaumcheon-gu, Korea). In order to distinguish between Psg22 and Psg25 and to ensure that there was no preferential amplification of any particular Psg, the above experiment was repeated using the primer set Psg-all2, which has the following sequences: Psg-all2F: 5'GTGTTGACAATCTGCCAGAGAATCTT; Psg-all2R: 5'CTCCTGGGTGACATTTTGGATC. PCR was performed in $50 \mu \mathrm{l}$ using $0.4 \mathrm{U}$ Accuzyme according to the manufacturer's instructions (Bioline). The following amplification protocol was used: denaturation at $95^{\circ} \mathrm{C}$ for $5 \mathrm{~min}$, amplification repeated for 40 cycles at $95^{\circ} \mathrm{C}$ for $30 \mathrm{~s}, 55^{\circ} \mathrm{C}$ for $30 \mathrm{~s}, 72{ }^{\circ} \mathrm{C}$ for $45 \mathrm{~s}$ and elongation cycle at $72{ }^{\circ} \mathrm{C}$ for $10 \mathrm{~min}$. An amplicon of $176 \mathrm{bp}$ was generated which was confirmed by gel electrophoresis on a standard $1.5 \%$ agarose gel as described above.

\section{Embryo transfers to CD9 - /- and CD9 +/+ mice}

Matings between CD9 heterozygous $(+/-)$ males and females were used to generate homozygous null $(-/-)$ females as test subjects and wild-type $(+/+)$ females as littermate controls on the same genetic background. $\mathrm{CD} 9+/+\left(\mathrm{B} 6 \mathrm{CBF}_{2}\right)$ embryos were produced, collected and transferred at the late morula or early blastocyst stage to the uterine horns of CD9 $+/+$ or CD9 $-/-$ pseudopregnant females as described (Nagy et al. 2003), with eight embryos being transferred to each uterine horn. Recipients were monitored daily for recovery, signs of pregnancy and pregnancy loss. Mice were killed at either E10 or E16, the uterus was removed and the number of successful and failed implantation sites was recorded.

\section{Results \\ Specificity of anti-Psg21 and anti-PSG1 antisera}

To determine the location of Psg protein during mouse placentation, a polyclonal antiserum was generated against recombinant Psg21. In parallel, human PSG1 protein and antiserum were also produced. On Western blot, recombinant PSG1 and Psg21 proteins had molecular weights of approximately $55 \mathrm{kDa}$ and $62 \mathrm{kDa}$ respectively, close to the predicted size for the glycosylated isoforms observed in vivo (Sorensen 1984, our unpublished observations). Using these recombinant proteins, anti-Psg21 and anti-PSG1 polyclonal antisera were produced in rabbits.

Using immunohistochemistry, Psg21 antiserum produced a pattern of staining on tissue sections of mouse placenta that was broadly consistent with expectations (see below). However, although it detected recombinant Psg21V5/His on Western blots, it did not detect Psg in mouse placental lysates or in maternal serum of pregnant mice. It also failed to detect recombinant Psg21 that was over-expressed in HeLa cells from a transfected pcDNA3.1 (Invitrogen Life Technologies) expression vector (data not shown). In contrast, the anti-human PSG1 antiserum worked effectively on Western blots, detecting bands consistent with endogenous PSG in human pregnant sera and placental lysates, and recombinant PSG1 and PSG9 over-expressed in HeLa cells from transfected pcDNA3.1 expression vectors (data not shown). These results were identical to those obtained using AB653, a commercially sourced anti-human PSG antibody raised against PSG purified from the urine of pregnant women. When tested against mouse placental tissues or transgenically over-expressed recombinant mouse Psg21 in HeLa cell lysates, PSG1 antiserum did not detect mouse Psg on Western blots.

To exclude the possibility of cross-reaction of the Psg21 antiserum to the widely expressed Ceacam proteins, which are closely related to Psg, the antiserum was tested by immunohistochemistry on mouse tissues that express Ceacam, but not Psg proteins including liver, colon, small intestine, testis, ovary, brain and non-pregnant uterus. 
The anti-Ceacam antibody, AgB10 (Kuprina et al. 1990), was used as a positive control (Fig. 1b). The Psg21 antiserum did not cross-react with Ceacam in bile canaliculi of liver (Fig. 1a), nor in any of the other tissues tested (data not shown). Pre-immune serum for the Psg21 antiserum was used as a negative control (Fig. 1C).

\section{Psg staining is associated with trophoblast and maternal vascular endothelium}

The expression of Psg in the developing mouse placenta was investigated by in situ hybridisation to mRNA using a digoxigenin-labelled Psg21 RNA probe, and by immunofluorescence and immunohistochemistry using Psg21 antiserum. Unexpectedly, in situ hybridisation did not detect Psg mRNA in giant cells between E8 and E11, which may reflect lack of sensitivity of our technology combined with relatively low levels of Psg mRNA in giant cells (see below). However, strong staining of spongiotrophoblast was evident at E14 to E16 (Fig. 2e). Psg protein was detected abundantly in giant cells from E8 to E11. Psg was distributed in vesicular structures, reminiscent of secretory granules, throughout the cytoplasm (Fig. 2a). However, at E15 there was only faint, diffuse Psg immunoreactivity in the cytoplasm of giant cells (Fig. 2b). Unexpectedly, the Psg21 antiserum did not detect Psg protein in the spongiotrophoblast of E14 and later stage placentas (Fig. 2C), in spite of the high levels of mRNA detected by in situ hybridisation.

Psg staining was observed lining the capillaries within the decidua of the implantation site from E8 to E11 using the anti-Psg21 antiserum. This staining was located predominantly on the antimesometrial aspect of the implantation site but also extended laterally towards the mesometrial pole. An antibody to CD31 (DeLisser et al. 1997, Newman 1997), an endothelial cell marker, was used to confirm this localisation (Fig. 3a-f). Staining for Psg was much less obvious at the mesometrial pole of the implantation site from E8 to E11 and was not seen in earlier implantation sites on E6 and E7.

\section{CD9 is expressed in maternal decidual tissues}

From the earliest post-implantation stages examined, CD9 completely surrounds the embryo within the implantation site, as determined by immunohistochemistry and immunofluorescence (Fig. 4A, a-c). CD9 expression is located on the cell surface and in the cytoplasm of maternal decidual tissue cells immediately surrounding the embryonic tissue. As pregnancy progresses, from E6 onwards, the intensity of CD9 staining increases and extends throughout the rest of the decidua, with a distinctly polarised pattern of expression (Fig. 4A, c, e-j). Mesometrially, CD9 expression is strongest lining the endothelial cells of capillaries and vascular spaces within the decidua (Fig. 4A, c, e, f), whereas antimesometrially, CD9 staining is observed throughout the tissue, with both the cytoplasm and surface of decidual stromal cells staining strongly (Fig. 4A, c, g, i, j). Subsequent to E12, there is reduced CD9 staining of the implantation site as the decidual tissue is reduced on the antimesometrial and lateral aspects of the implantation site; however, the endothelium of the maternal sinuses in the remaining mesometrial decidual tissue still exhibits strong CD9 staining (Fig. 4A, d). Staining is also observed in the uterine lumenal epithelium, uterine glandular epithelium, myometrium and in blood vessels cross-sectioned within the myometrium. To determine whether CD9 expression levels in decidual tissues are similar to those found in other tissues, Western blotting was carried out using decidual tissues from E8 to E11, and a range of adult tissues. There was no observable difference in expression levels between decidual tissues and other tissues (Fig. 4B).

\section{Co-localisation of CD9 and Psg in the placenta}

CD9 staining is present in several trophoblast cell types. From E8 onwards, CD9 staining is found both on the cell surface of primary trophoblast giant cells and also as an intense punctate distribution within the cytoplasm (Fig. 4A, I). Cell surface staining of secondary trophoblast giant cells is found from E10 onwards (Fig. 4A, h, k). The labyrinthine layer of the placenta also shows extensive CD9 staining, whereas staining is less intense in the spongiotrophoblast and even further reduced in glycogen cells (Fig. 4A, d).

Double immunoflouresence staining experiments with the anti-CD9 and anti-Psg21 antisera revealed that both proteins are found contemporaneously at several locations in fetal and maternal tissues. Psg and CD9 are found on the cell surface of primary and secondary trophoblast giant cells (Fig. 4A, h, k, I).
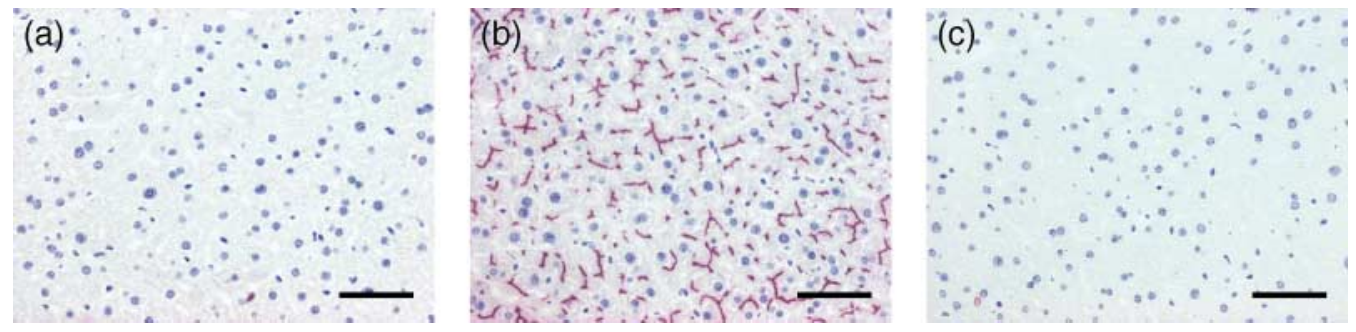

Figure 1 Immunohistochemistry of adult liver tissue sections, which express Ceacams, using (a) anti-Psg23 antiserum, (b) the anti-Ceacam antibody, AgB10, and (c) preimmune serum control for anti-Psg23 antiserum. Scale bar, $100 \mu \mathrm{m}$. 

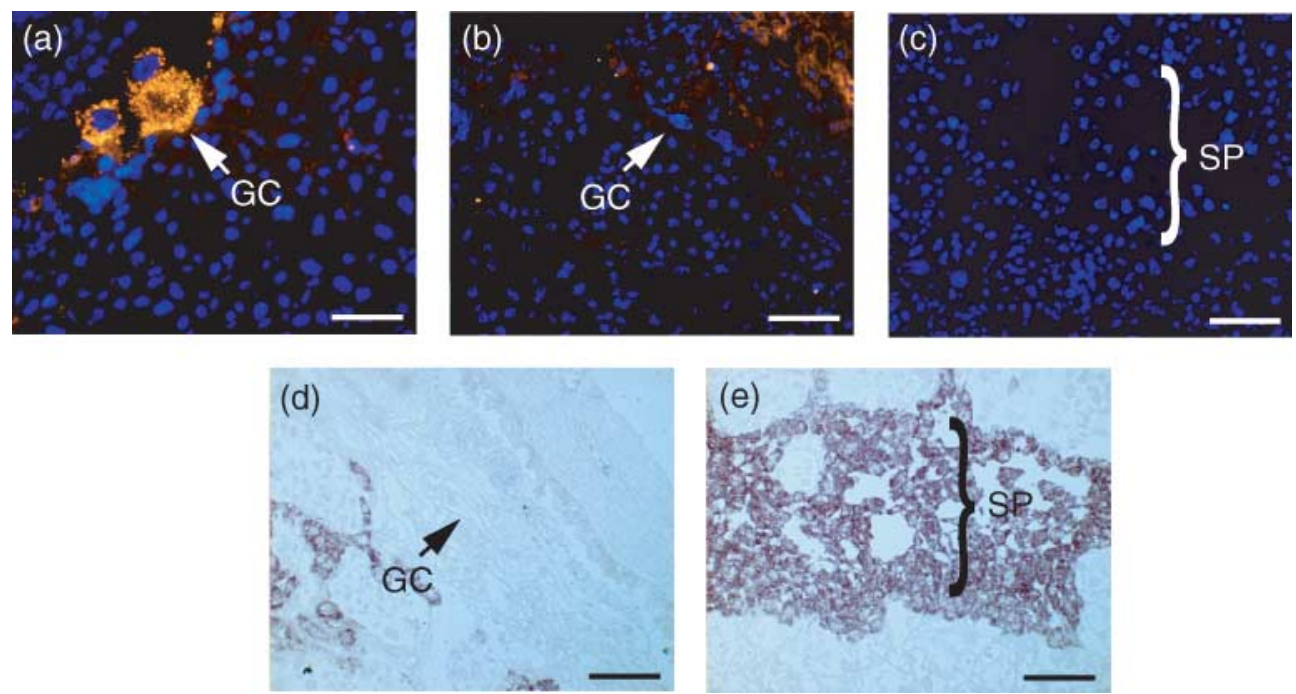

Figure 2 Immunofluorescent detection of Psg with anti-Psg23 antiserum in (a) E9 giant cells, (b) E15 giant cells, (c) E15 spongiotrophoblast (no staining observed). In situ hybridisation using digoxigenin-labelled Psg23 RNA probes in (d) E15 giant cells and (e) E15 spongiotrophoblast is shown. GC, giant cells; SP, spongiotrophoblast layer. Scale bar, $100 \mu \mathrm{m}$.

In the decidua, from the earliest observed expression of Psg (E8), there is evidence of co-localisation of Psg and CD9 in the endothelium of capillaries and vascular spaces within the decidua, which is evident until E11 (Fig. 4A, e-g, i, j). CD9 expression in the decidua starts at a much earlier stage (E6) and is both intense and ubiquitous.

\section{Psg expression levels exhibit tissue and developmental stage specificity}

Quantitative RT-PCR was used to determine the level of Psg gene transcripts in the trophoblast giant cells and in the spongiotrophoblast. PCR primers were designed to amplify a region in the $\mathrm{N}$ domain of all known mouse $P s g$ genes. Primer concentration was optimised to determine the lowest threshold cycle $(\mathrm{Ct})$ while minimising nonspecific amplification. This concentration was found to be $300 \mathrm{pmol}$ for the PsgF and PsgR primer set, and dissociation curves for the PCR product demonstrated a single specific peak indicating absence of non-specific amplification.

In trophoblast giant cells there is increased $P_{s g}$ expression between E8 and E11 (Fig. 5). Psg transcript levels double from E8 to E9 and from E9 to E10. In
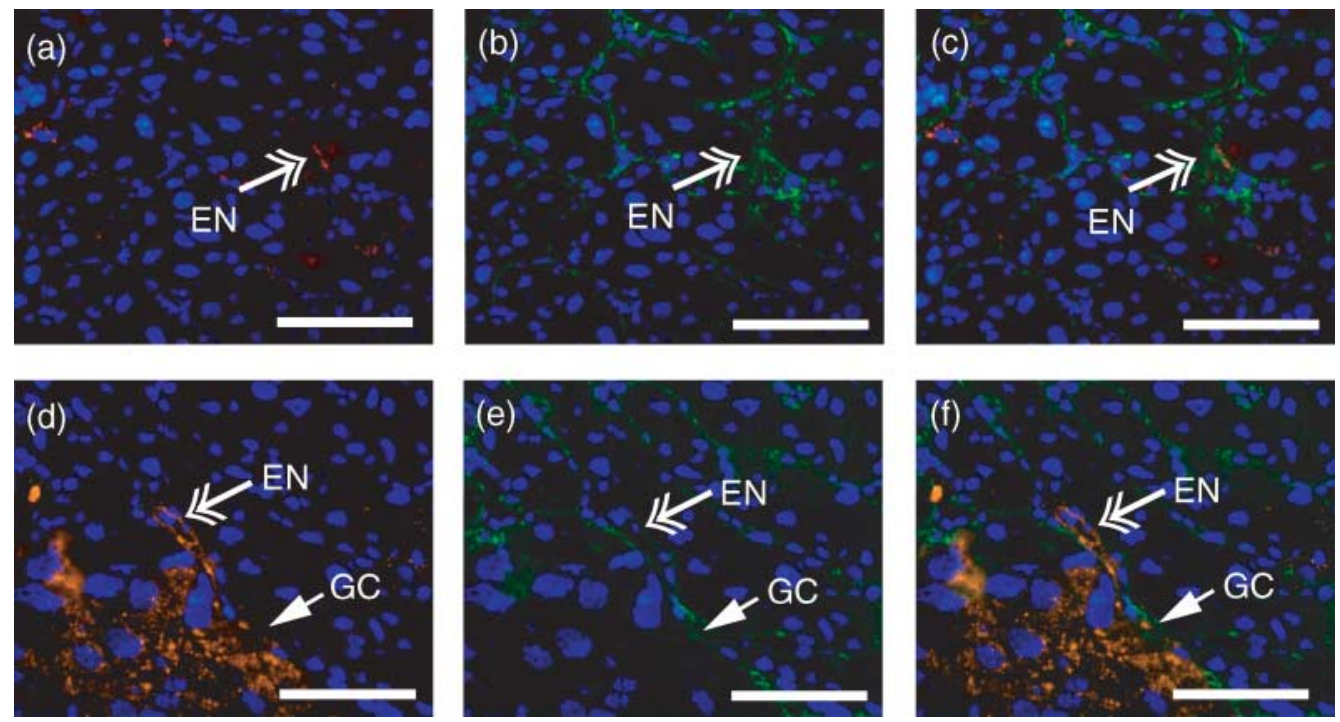

Figure 3 Double immunofluorescence detection of Psg (red) and CD31 endothelial cell marker (green) in decidual tissue surrounding the implantation site on E8 (a-c) and E10 (d-f). (a and d) Anti-Psg23 antiserum, (b and e) anti-CD31 antibody, (c and f) merged anti-Psg23 and antiCD31. EN, endothelium; GC, giant cell layer. Scale bar, $100 \mu \mathrm{m}$. 

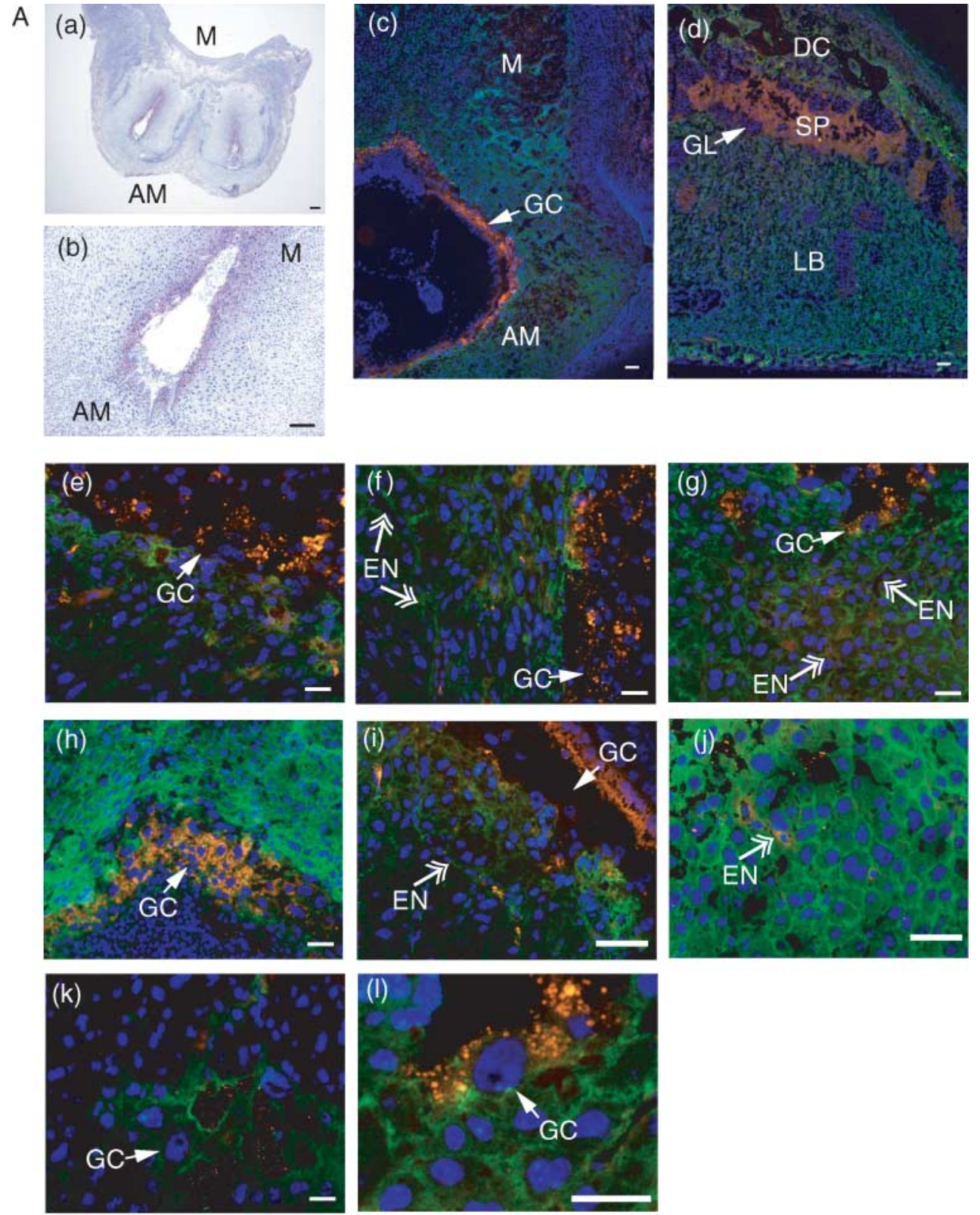

B

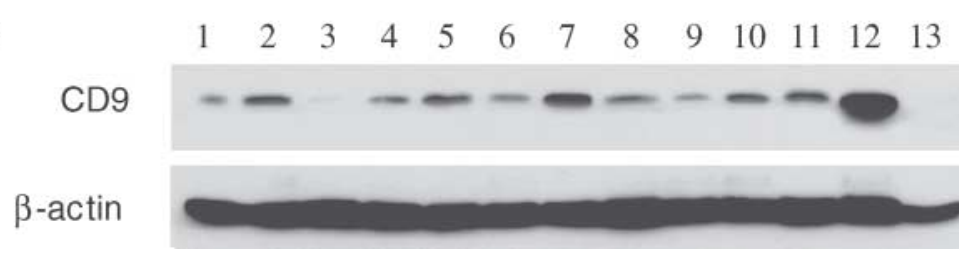

Figure 4 (A) (a-d) Immunohistochemical localisation of CD9 in E6 implantation site at low magnification: (a) scale bar $200 \mu \mathrm{m}$ and (b) scale bar $100 \mu \mathrm{m}$. Low power image of CD9 (green) and Psg (red) immunofluorescence on E10 (c), and CD9 (green) only on E15 (d); scale bars, $100 \mu \mathrm{m}$. (e-l) High magnification double immunofluorescence staining of Psg and CD9: (e and f) E8 lateral to mesometrial, (g) E8 antimesometrial, (h) E10 mesometrial, (i) E10 lateral, (j) E10 antimesometrial, and (k) E15 secondary giant cell. (I) Punctate Psg and CD9 staining in primary giant cell. Scale bars in (e-l), $50 \mu \mathrm{m}$. GL, glycogen cells; GC, giant cell layer; DC, decidua; SP, spongiotrophoblast layer; LB, labyrinthine layer; EN, endothelium; M, mesometrial aspect; AM, antimesometrial aspect. (B) Relative CD9 expression levels in mouse tissues. Lanes 1-4: E8, E9, E10 and E11 decidual tissues; lanes 5-8: E8, E9, E10 and E11 uterine tissues; lane 9: E10 ectoplacental cones; lane 10: E10 giant cells; lane 11: adult brain; lane 12: adult lung; lane 13: adult liver. $\beta$-Actin staining indicates approximately equal loading of lanes. In this instance, E10 decidual tissues (lane 3) exhibit low levels of CD9 expression. However, in other experiments, E10 CD9 expression was similar to other stages. 


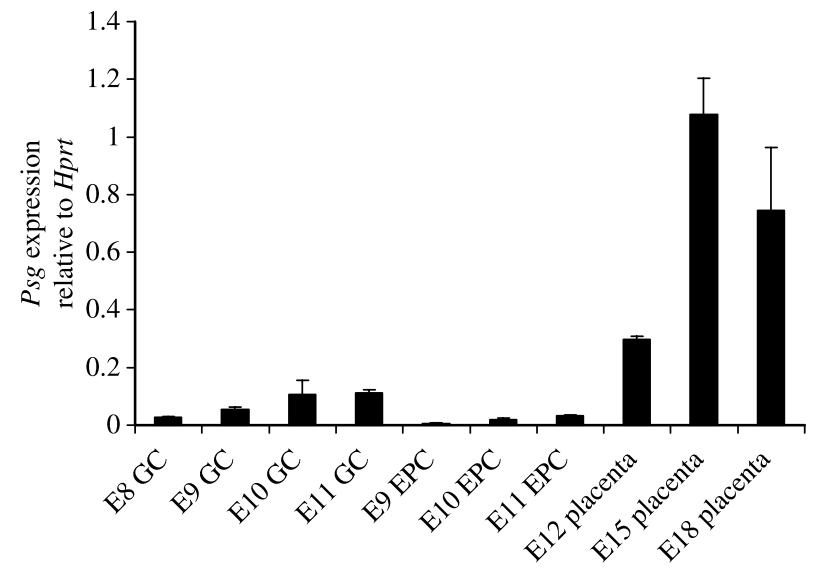

Figure 5 Quantification of $P_{s g}$ gene transcription relative to Hprt in giant cells on E8 to E11, ectoplacental cones on E9 to E11, and entire placentas on E12, E15, E18. Data are means \pm standard deviations, $n=2$ litters (cDNA was made from pooled mRNA from approximately six placentas per litter).

ectoplacental cone (EPC), there is a fivefold increase in Psg transcript levels between E9 and E11 (Fig. 5). However, absolute levels in the EPC are low, with E10 giant cells having approximately sixfold higher levels than E10 EPC. In dissected whole placenta samples (of which only the spongiotrophoblast compartment supports Psg gene transcription), Psg transcript levels increased fourfold from E12 to E15 (Fig. 5), and thereafter declined by approximately fifty per cent to E18.

\section{Psg22 is the most abundant Psg gene transcript in trophoblast giant cells}

Previous studies have shown that Psg21 and Psg23 gene transcripts together constitute the bulk of Psg gene expression in the spongiotrophoblast (Ball et al. 2004, McLellan et al. 2005a). To determine whether specific Psg gene transcripts similarly dominate in giant cells, $P s g$ transcripts were analysed in giant cells at four stages in development: E8, E9, E10 and E11. cDNA samples were amplified with degenerate primers designed to amplify all murine $P_{s g}$ gene transcripts (Psg16 - Psg32). Psg22 and Psg25 sequences are identical in the amplicon generated. Amplicons were subcloned into pSTBlue vector and eight of the resulting clones from each litter were sequenced in both directions for each developmental stage. As detailed in Table 1, the clones were overwhelmingly derived from either Psg22 or Psg25. Other Psg gene transcripts were represented at lower levels $(10 \%$ or less), and included Psg19, Psg24 and Psg29 at E8, Psg19 and Psg29 at E9, Psg29 at E10 and Psg29 at E11. The experiment was repeated using alternative degenerate PCR primers (Psg-all2) that allow Psg22 and Psg25 to be distinguished. Of the 10 clones derived from E10 giant cells, all were Psg22.
Table 1 Psg gene transcripts in CD1 trophoblast giant cells from E8-E11

\begin{tabular}{|c|c|c|c|}
\hline $\begin{array}{l}\text { Developmental } \\
\text { stage }\end{array}$ & $\begin{array}{l}\text { PCR } \\
\text { primers }\end{array}$ & $\begin{array}{l}\text { No. clones } \\
\text { sequenced }\end{array}$ & $\begin{array}{l}\text { Psg transcripts } \\
\text { present }(n)\end{array}$ \\
\hline E8 & PsgF\&R & 18 & $\begin{array}{l}\text { Psg22/25 (11) } \\
\text { Psg19(13) } \\
\text { Psg24 (1) } \\
\text { Psg29 (1) } \\
\text { Ceacam9 (2) }\end{array}$ \\
\hline E9 & PsgF\&R & 16 & $\begin{array}{l}\text { Psg22/25 (12) } \\
\text { Psg19 (1) } \\
\text { Psg29 (1) } \\
\text { Loc384557 (2) }\end{array}$ \\
\hline E10 & PsgF\&R & 16 & $\begin{array}{l}\text { Psg22/25 (15) } \\
\text { Psg29 (1) }\end{array}$ \\
\hline E11 & PsgF\&R & 17 & $\begin{array}{l}\text { Psg22/25 (14) } \\
\text { Psg29 (3) }\end{array}$ \\
\hline E10 & Psg-all2 & 10 & Psg22 (10) \\
\hline
\end{tabular}

\section{Maternal CD9 is not essential for successful pregnancy}

To determine whether maternally expressed CD9 is required for embryo implantation and pregnancy, wildtype embryos of the $\mathrm{B}_{6} \mathrm{CBF}_{2}$ strain were transferred to wild-type $(\mathrm{CD} 9+/+)$ and null $(\mathrm{CD} 9-/-)$ pseudopregnant females. To control for genetic background effects on fertility, both wild-type and null recipient females were derived from offspring of the $\mathrm{CD} 9+/-\times \mathrm{CD} 9+/-$ intercross on the C57BL/6 genetic background.

CD9 was not required for a successful pregnancy up to E16, the latest stage at which embryo recipients were examined (Table 2). No difference was observed between CD9 $+/+$ and CD9 $-/-$ recipients with respect to number of implantations, number of resorptions, developmental stage or gross morphology of embryos or placentas.

\section{Discussion}

We analysed the expression of the mouse Psg and CD9 proteins during pregnancy to determine whether their expression patterns are consistent with mutual interactions in vivo. We also sought to determine whether specific $P_{s g}$ genes are expressed at different developmental stages, and to attempt to assess the likely importance of Psg/CD9 interactions for successful pregnancy.

Psg expression and localisation were analysed using a combination of in situ hybridisation to mRNA and immunohistochemistry and immunofluorescence. At the time of this study, a specific anti-mouse Psg antibody was not

Table 2 Embryo transfer to CD9 + /+ (wildtype) and CD9 - /(null) mice.

\begin{tabular}{lcc}
\hline & CD9 $+/+$ & CD9 -/- \\
\hline Total recipient females & 14 & 17 \\
Recipients pregnant at E10 or E16 & 10 & 12 \\
Mean implantation sites/pregnancy & 7.33 & 7.16 \\
\hline
\end{tabular}

Reproduction (2006) 131 721-732 
available, and stocks of commercially available antihuman PSG (AB653) were low. We therefore produced antisera to recombinant mouse Psg21 and human PSG1. Our anti-PSG1 antiserum behaved predictably on Western blots of human tissues, comparable to AB653. However, the anti-Psg21 antiserum did not detect endogenous mouse Psg on Western blot, but was successfully used for immunohistochemistry of trophoblast giant cells. Unexpectedly, however, the Psg21 antibody did not detect Psg in spongiotrophoblast, the major site of $P s g$ gene transcription. Potential explanations for this anomaly are that the Psg21 antiserum recognises a restricted set of epitopes that may only be present in the limited set of Psg genes expressed in giant cells (see below), or there may be masking of relevant epitopes (e.g. by glycosylation) in spongiotrophoblast. Alternatively, in spite of high levels of Psg mRNA, there may be low steady state levels of Psg protein due to translational regulation or rapid turnover. An alternative explanation, given our failure to validate our anti-Psg21 antiserum to the same standard as our antiPSG1 antiserum, is that staining of giant cells is artefactual. While we cannot formally exclude this possibility subject to the generation of further anti-mouse Psg antibodies, we consider it unlikely because staining is confined to giant cells, which express Psg mRNA, and to maternal vasculature expressing the Psg receptor CD9 (see below), which would be directly exposed to secreted Psg.

Subject to the aforementioned caveat, Psg staining was observed on the endothelial lining of vascular spaces in the decidual tissue surrounding the implantation site from E8 to E11. Since there was no evidence from this or previous studies of $P s g$ gene expression in endothelium of decidual tissues, we conclude that this staining may represent secreted Psg from fetal tissues, which becomes associated with maternal vascular endothelium. There is extensive angiogenesis and vascular remodelling associated with pregnancy, and trophoblast giant cells produce a complex array of angiogenic and anti-angiogenic and vasoactive compounds (Cross et al. 2002). Currently, Psg are thought to regulate the maternal immune system during pregnancy; however, the evidence in support of this hypothesis has largely been generated in vitro using immune cell models (Wessells et al. 2000, Snyder et al. 2001, Motran et al. 2003), and there remains the possibility that different Psg proteins encode different functions. One interpretation of our finding that Psg may associate with maternal vasculature is that some Psg may exhibit vasoactivity or angiogenesis-related functions.

Using immunohistochemistry and immunofluorescence, we found that CD9 is widely expressed in the fetal compartments of the placenta and in maternal decidual and other uterine tissues throughout pregnancy. CD9 is evident both intracellularly and on the cell surface of giant cells from E8 to E11. Rodent trophoblast giant cells are analogous to extravillous cytotrophoblast cells of the human placenta; both are polyploid and invasive, and have similar patterns of trophoblast cell subtype-specific gene expression (Hemberger \& Cross 2001). In the human placenta, the intensity of CD9 expression in the extravillous trophoblast cells differs between early pregnancy and term: expression in the extravillous trophoblast (EVT) invading the endometrium in early pregnancy is weak, but in the placental bed and chorionic laevae of the term placenta (where EVTs have ceased invasion into the endometrium) expression is intense (Hirano et al. 1999). This suggests that human CD9 might have a role in inhibiting cell invasion; however, from our data, we cannot confidently propose an analogous function for mouse CD9. From E13 onwards, CD9 staining is found in both the spongiotrophoblast and the labyrinth; in the latter case staining is predominantly associated with the cells lining the vascular channels.

Staining of decidual tissues was particularly intense, with evidence for both cytoplasmic and cell surface staining from E6. The significance of the different distributions of CD9 staining of decidual cells on the mesometrial and antimesometrial aspects of the implanted embryo at later stages is unclear: the intense staining of decidual cells on the antimesometrial aspect is particularly striking, whereas on the mesometrial aspect, staining was mostly confined to the surface of endothelial cells lining vascular spaces. The strong cytoplasmic staining of CD9 in decidual cells is somewhat unusual; however the overall magnitude of CD9 staining in decidual cells is similar to other tissues, as judged by Western blot. CD9 is generally found on the cell surface; however, occasionally, it is located intracellularly e.g. in eosinophils and platelets, where it is stored pending transfer to the cell surface upon activation (Fernvik et al. 1995, Brisson et al. 1997).

In summary, our findings show that there is considerable scope for secreted Psg to interact with maternal CD9. However, our results suggest that the bulk of CD9 expression in pregnancy is not associated with immune cells but, rather, with maternal decidual and vascular tissues. Our related finding of an association of Psg and vascular endothelium supports a scenario whereby Psg secreted from fetal tissues interacts with CD9 on the maternal vasculature. However, whether this putative interaction would result in the activation of signalling pathways relevant to endothelial cell function is unclear. An alternative scenario, based on the concept of maternal-fetal conflict (Moore \& Haig 1991, Haig 1993), is that maternal CD9 expressed on vascular endothelium acts as a 'sink' or decoy receptor for Psg, thereby reducing the amount of Psg available for interacting with maternal immune cells. In addition, we add the caveat that, because of the widespread expression of CD9 in maternal tissues, the observed co-localisation with Psg could be coincidental. Confirmation of putative functional interactions between these proteins at overlapping sites in maternal tissues will therefore require further analysis.

The independent expansion of primate and rodent PSG gene families in evolution suggests convergence of function (McLellan et al. 2005b). However, a previous 
semi-quantitative study of $P_{s g}$ gene expression in mouse pregnancy indicated that different family members exhibit different expression levels between E11 and E18, suggesting the possibility of divergent functions, at least within the mouse Psg family (McLellan et al. 2005a). To investigate this possibility further, we analysed the expression profile of $P_{s g}$ genes in the giant cells and spongiotrophoblast using quantitative methods. The earliest that Psg transcripts have been detected in the murine placenta is E6.5 in trophoblast giant cells (Finkenzeller et al. 2003). It is difficult to isolate cDNA from specific embryonic tissues at this stage, so our analysis began at E8. First, we analysed levels of total Psg gene expression using PCR primers designed to amplify transcripts from all $P s g$ genes. Total levels of $P s g$ transcripts in giant cells increased between E8 and E11, but remained considerably lower (approximately tenfold) than in whole placenta from mid to late gestation. This is consistent with our failure to detect Psg transcripts in giant cells using in situ hybridisation. Very low levels of Psg transcription were also detected in dissected ectoplacental cones; this may represent contamination from adherent giant cells or, alternatively, the earliest manifestation of differentiating spongiotrophoblast from late E10 and E11. Psg22 was overwhelmingly the most abundant $P_{s g}$ transcript in giant cells between E8 and E11, consistent with a previous study that did not detect Psg22 expression from E11 onwards, when the giant cells form a progressively reduced proportion of placental tissues (McLellan et al. 2005a). Psg22 may be expressed virtually exclusively in giant cells and may encode a specific or additional function not associated with other Psg proteins. In this context, it would be interesting to determine whether Psg22, like Psg17 and Psg19, binds CD9.

In spite of the widespread expression of CD9 in the pregnant uterus, the transfer of wild-type embryos to CD9deficient females resulted in comparable pregnancy rates to embryos transferred to wild-type females, suggesting that maternal CD9 expression is not essential for successful implantation or maintenance of pregnancy. If CD9 represented the sole receptor for secreted fetal Psg, the CD9 null female would represent a surrogate Psg null mutant, with important implications for understanding the significance of Psg in pregnancy. However, it is currently unclear whether all mouse Psg bind CD9, or whether those that do, do so exclusively. In this context, it is noteworthy that human PSG do not bind CD9, but nevertheless induce expression of a similar range of cytokines to mouse Psg from monocytes (Ha et al. 2005). The elucidation of Psg function in mouse pregnancy will therefore require further developmental and biochemical studies using techniques such as gene targeting to assess the importance of specific Psg genes, and proteomics to collate the full spectrum of putative Psg/receptor interactions on trophoblast cells, maternal vascular endothelium and maternal immune cells.

\section{Acknowledgements}

We thank Dr C Boucheix, Institut National de la Santé et de la Recherche Médicale (INSERM), France, for a gift of CD9 null mice, and Dr NI Kuprina, Laboratory of Immunochemistry, All-Union Cancer Research Centre, Moscow, USSR, for the AgB10 hybridoma. This work was supported by the Irish Higher Education Authority Programme for Research in Third Level Institutions funded under the National Development Plan, and an Irish Health Research Board/Wellcome Trust 'New Blood' Research Fellowship to T Moore. The authors declare that there is no conflict of interest that would prejudice the impartiality of this scientific work.

\section{References}

Ball M, McLellan A, Collins B, Coadwell J, Stewart F \& Moore T 2004 An abundant placental transcript containing an IAP-LTR is allelic to mouse pregnancy-specific glycoprotein 23 (Psg21): cloning and genetic analysis. Gene 325 103-113.

Bohn H \& Weinmann E 1976 [Antifertility effect of an active immunization of monkeys with human pregnancy-specific beta 1-glycoprotein (SP1) (author's transl)]. Archiv fur Gynakologie 221 305-312.

Boucheix C \& Rubenstein E 2001 Tetraspanins. Cell Molecular Life Science 58 1189-1205.

Brisson C, Azorsa DO, Jennings LK, Moog S, Cazenave JP \& Lanza F 1997 Co-localization of CD9 and GPllb-Illa (alpha Ilb beta 3 integrin) on activated platelet pseudopods and alpha-granule membranes. Histochemistry Journal 29 153-165.

Brummendorf T \& Rathjen FG 1994 Cell adhesion molecules. 1. Immunoglobulin superfamily. Protein Profile 1 951-1058.

Cross JC, Hemberger M, Lu Y, Nozaki T, Whiteley K, Masutani M \& Adamson SL 2002 Trophoblast functions, angiogenesis and remodeling of the maternal vasculature in the placenta. Molecular and Cellular Endocrinology 187 207-212.

DeLisser HM, Christofidou-Solomidou M, Strieter RM, Burdick MD, Robinson CS, Wexler RS, Kerr JS, Garlanda C, Merwin JR, Madri JA \& Albelda SM 1997 Involvement of endothelial PECAM-1/CD31 in angiogenesis. American Journal of Pathology 151 671-677.

Ellerman DA, Ha C, Primakoff P, Myles DG \& Dveksler GS 2003 Direct binding of the ligand PSG17 to CD9 requires a CD9 site essential for sperm-egg fusion. Molecular Biology of Cell 14 5098-5103.

Fernvik E, Hallden G, Hed J \& Lundahl J 1995 Intracellular and surface distribution of CD9 in human eosinophils. Acta Pathologica, Microbiologica et Immunologica Scandinavica 103 699-706.

Fialova L, Kohoutova B, Peliskova Z, Malbohan I \& Mikulikova L 1991 [Serum levels of trophoblast-specific beta-1-globulin (SP1) and alpha-1-fetoprotein (AFP) in pregnant women with rheumatoid arthritis]. Ceskoslovenská Gynekologie 56 166-170.

Finkenzeller D, Fischer B, Lutz S, Schrewe H, Shimizu T \& Zimmermann W 2003 Carcinoembryonic antigen-related cell adhesion molecule 10 expressed specifically early in pregnancy in the decidua is dispensable for normal murine development. Molecular and Cellular Biology 23 272-279.

Ha CT, Waterhouse R, Wessells J, Wu JA \& Dveksler GS 2005 Binding of pregnancy-specific glycoprotein 17 to CD9 on macrophages induces secretion of IL-10, IL-6, PGE 2 , and TGF- $\beta 1$. Journal of Leukocyte Biology 77 948-957.

Haig D 1993 Genetic conflicts in human pregnancy. Quarterly Review of Biology 68 495-532.

Han E, Phan D, Lo P, Poy MN, Behringer R, Najjar SM \& Lin SH 2001 Differences in tissue-specific and embryonic expression of mouse Ceacam1 and Ceacam2 genes. Biochemistry Journal 355 417-423. 
Harris SJ, Anthony FW, Jones DB \& Masson GM 1984 Pregnancyspecific-beta 1-glycoprotein: effect on lymphocyte proliferation in vitro. Journal of Reproductive Immunology 6 267-270.

Hau J, Gidley-Baird AA, Westergaard JG \& Teisner B 1985 The effect on pregnancy of intrauterine administration of antibodies against two pregnancy-associated murine proteins: murine pregnancy-specific beta 1-glycoprotein and murine pregnancy-associated alpha 2-glycoprotein. Biomedica et Biochimica Acta 44 1255-1259.

Hemberger M \& Cross JC 2001 Genes governing placental development. Trends in Endocrinological Metabolism 12 162-168.

Hirano T, Higuchi T, Katsuragawa H, Inoue T, Kataoka N, Park KR, Ueda M, Maeda M, Fujiwara H \& Fujii S 1999 CD9 is involved in invasion of human trophoblast-like choriocarcinoma cell line, BeWo cells. Molecular Human Reproduction 5 168-174.

Kromer B, Finkenzeller D, Wessels J, Dveksler G, Thompson J \& Zimmermann W 1996 Coordinate expression of splice variants of the murine pregnancy-specific glycoprotein (PSG) gene family during placental development. European Journal of Biochemistry 242 280-287.

Kuprina NI, Baranov VN, Yazova AK, Rudinskaya TD, Escribano M, Cordier J, Gleiberman AS \& Goussev Al 1990 The antigen of bile canaliculi of the mouse hepatocyte: identification and ultrastructural localization. Histochemistry 94 179-186.

Lei KJ, Sartwell AD, Pan CJ \& Chou JY 1992 Cloning and expression of genes encoding human pregnancy-specific glycoproteins. Journal of Biological Chemistry 267 16371-16378.

Lin TM, Halbert SP \& Spellacy WN 1974 Measurement of pregnancy-associated plasma proteins during human gestation. Journal of Clinical Investigation 54 576-582.

McLellan AS, Fischer B, Dveksler G, Hori T, Wynne F, Ball M, Okumura K, Moore T \& Zimmermann W 2005a Structure and evolution of the mouse pregnancy-specific glycoprotein (Psg) gene locus. BMC Genomics 64.

McLellan AS, Zimmermann W \& Moore T 2005b Conservation of pregnancy-specific glycoprotein (PSG) N-domains following independent expansions of the gene families in rodents and primates. BMC Evolutionary Biology 539.

Maecker HT, Todd SC \& Levy S 1997 The tetraspanin superfamily: molecular facilitators. FASEB Journal 11 428-442.

Majumdar S, Bapna BC, Mapa MK, Gupta AN, Devi PK \& Subrahmanyam D 1982 Pregnancy-specific proteins: suppression of in vitro blastogenic response to mitogen by these proteins. International Journal of Fertility 27 66-69.

Moore T \& Haig D 1991 Genomic imprinting in mammalian development: a parental tug-of-war. Trends in Genetics 7 45-49.

Motran CC, Diaz FL, Montes CL, Bocco JL \& Gruppi A 2003 In vivo expression of recombinant pregnancy-specific glycoprotein 1a induces alternative activation of monocytes and enhances Th2-type immune response. European Journal of Immunology 33 3007-3016.

Nagy A, Gertsenstein M, Vintersten K \& Behringer R 2003 In Manipulating the Mouse Embryo: A Laboratory Manual. Cold Spring Harbor, New York: CSHL Press.

Newman PJ 1997 The biology of PECAM-1. Journal of Clinical Investigation 100 S25-S29.

Rebstock S, Lucas K, Weiss M, Thompson J \& Zimmermann W 1993 Spatiotemporal expression of pregnancy-specific glycoprotein gene rnCGM1 in rat placenta. Developmental Dynamics 198 171-181.
Repina MA, Blagoslovenskii GS, Gnilevskaia ZU \& Ivanova LV 1989 [The effect of specific trophoblastic beta 1-glycoprotein on changes in the cellular link of immunity in infected abortion]. Akusherstvo i Ginekologiia 12 47-50.

Snyder SK, Wessner DH, Wessells JL, Waterhouse RM, Wahl LM, Zimmermann W \& Dveksler GS 2001 Pregnancy-specific glycoproteins function as immunomodulators by inducing secretion of IL-10, IL-6 and TGF-beta1 by human monocytes. American Journal of Reproduction and Immunology 45 205-216.

Sorensen S 1984 Pregnancy-"specific" beta 1-glycoprotein (SP1): purification, characterization, quantification and clinical application in malignancies (a review). Tumour Biology 5 275-302.

Teglund S, Olsen A, Khan WN, Frangsmyr L \& Hammarstrom S 1994 The pregnancy-specific glycoprotein (PSG) gene cluster on human chromosome 19: fine structure of the 11 PSG genes and identification of 6 new genes forming a third subgroup within the carcinoembryonic antigen (CEA) family. Genomics 23 669-684.

Thompson J, Koumari R, Wagner K, Barnert S, Schleussner C, Schrewe H, Zimmermann W, Muller G, Schempp W \& Zaninetta D 1990 The human pregnancy-specific glycoprotein genes are tightly linked on the long arm of chromosome 19 and are coordinately expressed. Biochemical and Biophysical Reseach Communications $167848-859$.

Waterhouse R, Ha C \& Dveksler GS 2002 Murine CD9 is the receptor for pregnancy-specific glycoprotein 17. Journal of Experimental Medicine 195 277-282.

Wessells J, Wessner D, Parsells R, White K, Finkenzeller D, Zimmermann W \& Dveksler G 2000 Pregnancy specific glycoprotein 18 induces IL-10 expression in murine macrophages. European Journal of Immunology 30 1830-1840.

Zebhauser R, Kammerer R, Eisenried A, McLellan A, Moore T \& Zimmermann W 2005 Identification of a novel group of evolutionary conserved members within the rapidly diverging murine Cea family. Genomics 86 566-580.

Zhou GQ \& Hammarstrom S 2001 Pregnancy-specific glycoprotein (PSG) in baboon (Papio hamadryas): family size, domain structure, and prediction of a functional region in primate PSGs. Biology of Reproduction 64 90-99.

Zhou GQ, Baranov V, Zimmermann W, Grunert F, Erhard B, Mincheva-Nilsson L, Hammarstrom S \& Thompson J 1997 Highly specific monoclonal antibody demonstrates that pregnancyspecific glycoprotein (PSG) is limited to syncytiotrophoblast in human early and term placenta. Placenta 18 491-501.

Zimmermann W, Weiss M \& Thompson JA 1989 cDNA cloning demonstrates the expression of pregnancy-specific glycoprotein genes, a subgroup of the carcinoembryonic antigen gene family, in fetal liver. Biochemical and Biophysical Research Communications $1631197-1209$

Received 4 July 2005

First decision 22 August 2005

Revised manuscript received 30 November 2005

Accepted 16 December 2005 